University of Wollongong

Research Online

Faculty of Engineering and Information

Faculty of Engineering and Information

Sciences - Papers: Part B

Sciences

2019

Three-dimensional measurement with reflection suppression based on high-dynamic range images

Limei Song

Tianjin Polytechnic University, lilymay1976@126.com

Yuan Ru

Tianjin Polytechnic University

Yangang Yang

Tianjin University of Technology And Education

Qinghua Guo

University of Wollongong, qguo@uow.edu.au

Xinjun Zhu

Tianjin Polytechnic University

See next page for additional authors

Follow this and additional works at: https://ro.uow.edu.au/eispapers1

Part of the Engineering Commons, and the Science and Technology Studies Commons

Research Online is the open access institutional repository for the University of Wollongong. For further information contact the UOW Library: research-pubs@uow.edu.au 


\title{
Three-dimensional measurement with reflection suppression based on high- dynamic range images
}

\author{
Abstract \\ To deal with the three-dimensional (3-D) point cloud loss caused by object reflection in the active fringe \\ projection 3-D measurement, an active reflection suppression method for 3-D measurement is proposed. \\ The method employs high-dynamic range images obtained by multiple exposure image fusion and a \\ three-wavelength phase-shift profilometry method to achieve high-precision 3-D measurement of \\ reflective objects. Experimental results show that, compared to traditional 3-D measurement methods, the \\ proposed one can more effectively handle reflections thereby avoiding 3-D point cloud loss in the \\ measurement of reflective objects. \\ Disciplines \\ Engineering | Science and Technology Studies

\section{Publication Details} \\ L. Song, Y. Ru, Y. Yang, Q. Guo, X. Zhu \& J. Xi, "Three-dimensional measurement with reflection \\ suppression based on high-dynamic range images," Journal of Electronic Imaging, vol. 28, (1) pp. \\ 013010-1-013010-10, 2019.
}

\section{Authors}

Limei Song, Yuan Ru, Yangang Yang, Qinghua Guo, Xinjun Zhu, and Jiangtao Xi 


\title{
Electronnic Imaging
}

\section{Three-dimensional measurement with reflection suppression based on high- dynamic range images}

\author{
Limei Song \\ Yuan $\mathrm{Ru}$ \\ Yangang Yang \\ Qinghua Guo \\ Xinjun Zhu \\ Jiangtao Xi
}




\title{
Three-dimensional measurement with reflection suppression based on high-dynamic range images
}

\author{
Limei Song, ${ }^{a}$ Yuan Ru, ${ }^{a}$ Yangang Yang, ${ }^{b}$ Qinghua Guo, ${ }^{a, c, *}$ Xinjun Zhu, ${ }^{a}$ and Jiangtao $\mathrm{Xi}^{\mathrm{c}}$ \\ ${ }^{a}$ Tianjin Polytechnic University, Key Laboratory of Advanced Electrical Engineering and Energy Technology, Tianjin, China \\ ${ }^{\mathrm{b}}$ Tianjin University of Technology and Education, School of Mechanical Engineering, Tianjin, China \\ 'University of Wollongong, School of Electrical, Computer, and Telecommunications Engineering, Wollongong, Australia
}

\begin{abstract}
To deal with the three-dimensional (3-D) point cloud loss caused by object reflection in the active fringe projection 3-D measurement, an active reflection suppression method for 3-D measurement is proposed. The method employs high-dynamic range images obtained by multiple exposure image fusion and a three-wavelength phase-shift profilometry method to achieve high-precision 3-D measurement of reflective objects. Experimental results show that, compared to traditional 3-D measurement methods, the proposed one can more effectively handle reflections thereby avoiding 3-D point cloud loss in the measurement of reflective objects. () 2019 SPIE and IS\&T [DOI: 10.1117/1.JEI.28.1.013010]

Keywords: multiple exposure image fusion; high-dynamic range image; 3-D reconstruction; reflection suppression.

Paper 180813 received Sep. 19, 2018; accepted for publication Dec. 27, 2018; published online Jan. 22, 2019.
\end{abstract}

\section{Introduction}

Noncontact three-dimensional (3-D) measurement is widely used in quality inspection and process control because it is convenient to use and has high measurement accuracy. Active 3-D measurement technology normally has high precision and reliability, which has a variety of applications, such as heritage conservation, industrial production, and biomedical science. ${ }^{1,2}$ However, the mirror reflection of highly reflective objects can lead to camera saturation and thereby the loss of 3-D point cloud of the reflective parts. ${ }^{3}$ For example, Figs. 1(a) and 1(b) show an image of a plastic cylinder and the corresponding captured fringe pattern image. Due to the diffuse reflection on the surface, the camera can capture a clear fringe pattern image. In contrast, Fig. 1(c) shows the image of the shaft connector, and Fig. 1(d) shows a fringe pattern image captured by the camera with the same exposure time as Fig. 1(b), where we can see the severe mirror reflection. At present, spraying developer is often used in industry to solve this problem, but the method is time consuming and the precision is subject to the thickness of powder spraying.

In recent years, several methods are proposed to measure 3-D data in the presence of interreflections. Phase-shifting fringe projection can be combined with multiview stereo to avoid measurement error caused by diffuse reflection of object surface. ${ }^{4}$ Three-wavelength phase-shift profilometry (TWPSP) is an effective method that can suppress the phase disturbance caused by diffuse reflections. In this method, the phase disturbance caused by diffuse reflection is same from different views, and the phase is only used to establish the relationship between the cameras. However, this method is not suitable for measuring glossy surfaces with specular reflections, because specular reflection changes with different views.

The investigation on reflection suppression has attracted much attention. In order to suppress specular reflection in fringe pattern images, Umeyama and Godin ${ }^{5}$ proposed to suppress object reflection by adding polarization devices in 3-D imaging system. This method is simple to operate and effective in reflection suppression. However, it requires additional system complexity and weakens the light intensity of the collected images, thus affecting the accuracy of measurement. Waddington and Kofman ${ }^{6}$ proposed to handle reflection by correcting sine fringes. The intensity of the fringe pattern is adaptively adjusted, so that the maximum input gray level of the camera is kept within the normal acquisition range to avoid camera saturation. Zhang et al. ${ }^{7}$ used the point diffusion function and the camera corresponding function to adjust the intensity of the projection stripe to prevent the camera from overexposure. It can achieve better measurement results for different scenes. Babaie and Farahi ${ }^{8}$ and Babaie ${ }^{9}$ put forward a reflection elimination method by controlling the projection fringe intensity based on feedback. It can get high-dynamic range stripes. However, these methods may produce brightness and color distortion because spatial consistency is not fully considered in the fusion process.

In order to obtain better fringe images, Zhang et al. ${ }^{10}$ analyzed the features of phase-shifting fringe images and then produced the final fringe images from multiexposure images pixel-by-pixel by selecting the brightest but unsaturated corresponding pixels from one exposure. Liu et al. ${ }^{11}$ first set the minimum and maximum precision gray can be measured by the experiment. Then the mask image sequence is calculated, and finally each pixel of the edge image is generated by selecting the brightest within the setting range of the corresponding pixel from one set of fringe images. Jiang et al. ${ }^{12}$ fused the original fringe patterns acquired with different exposure times and illumination intensity to generate a synthetic fringe image. However, these methods rely on multiple exposures with different system configurations, which are mainly obtained through the offline calculation and cannot automatically adjust the configuration for different scenes. 


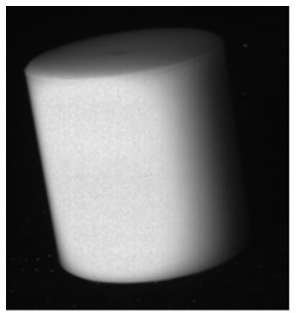

(a)

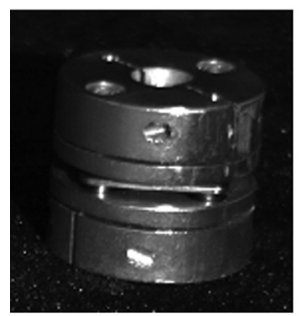

(c)

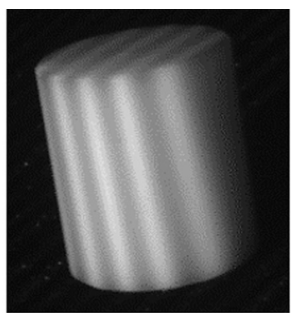

(b)

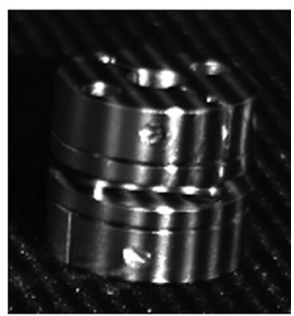

(d)
Fig. 1 The images of objects with different surface materials and the corresponding captured fringe pattern images: (a) a standard plastic cylinder, (b) a fringe pattern image of the plastic cylinder with exposure time $=80 \mathrm{~ms}$, (c) an aluminum alloy shaft connector, and (d) a fringe pattern image of the metal shaft connector with exposure time $=80 \mathrm{~ms}$.

To avoid the shortcomings of the above methods, a 3-D measurement reflection suppression method based on highdynamic range image is proposed. The method adaptively selects exposure time for different scenes and adaptively fuses multiple images under different exposure times to obtain high-dynamic range phase-shifting fringe images, thus achieving to suppress reflection. In order to obtain high-quality 3-D point cloud data, an improved TWPSP method is proposed, using the high-dynamic range phaseshifting fringe images, a full 3-D point cloud data of the reflective object is obtained.

The remainder of this paper is organized as follows. Section 2 elaborates the principles of the proposed multiple exposure image fusion (MEIF) method. In Sec. 3, the improved TWPSP method and the process of 3-D measurement with reflection suppression are detailed. Section 4 provides experimental results and analyses, and Sec. 5 concludes this paper.

\section{MEIF Method}

For reflective images, the light intensity is generally $>105 \mathrm{~cd} / \mathrm{m}^{2}$, while the dynamic range of most current CMOS and CCD sensor imaging is about $60 \mathrm{~dB}$. Therefore, the root cause of the reflection issue in 3-D measurement is the insufficient dynamic measurement range of cameras. ${ }^{13,14}$ Because of the limitation of the existing manufacturing process, it is difficult to improve cameras by hardware. In this paper, image fusion method is used to obtain a high-dynamic range image, thereby circumventing the problem of insufficient measurement range of cameras. Multiple image fusion is involved in the proposed method. In order to reduce the time of the whole reconstruction process, pixel fusion is adopted for adaptive fusion of multiple images with different exposure times.

\subsection{Selection of Image Fusion Weights}

For reflective objects, there are overexposure and underexposure areas in the acquired images, and images contain different effective information under different exposure times. ${ }^{12}$ Therefore, image exposure intensity factor $B_{k}(x, y)$ is a key factor of image fusion. In order to assign larger fusion weights to the pixels with moderate exposure time in the image, and to assign smaller fusion weights to the pixels with excessive exposure time and insufficient exposure time, the Gauss equation is selected to assign fusion weights to each pixel in the image. Assuming that the $k$ 'th collected gray normalized exposure image is $I_{k}^{\text {gray }}(x, y)(k=1,2, \ldots, N)$, and $N$ is the camera exposure time, then $B_{k}(x, y)$ can be calculated as

$$
\begin{aligned}
& B_{k}(x, y) \\
& \quad=\left\{\begin{array}{l}
e^{\frac{-\left[t_{k}^{\text {gray }}(x, y)-\mu(x, y)\right]^{2}}{2 \sigma^{2}}}, T_{L}<I_{k}^{\text {gray }}(x, y)<1-T_{L} \\
0,0 \leq I_{k}^{\text {gray }}(x, y) \leq T_{L} \text { or } 1-T_{L} \leq I_{k}^{\text {gray }}(x, y) \leq 1
\end{array},\right.
\end{aligned}
$$

with

$\mu(x, y)=0.5+0.3 *\left[\frac{1}{N} \sum_{k=1}^{N} I_{k}^{\text {gray }}(x, y)-0.5\right]$,

where $x$ and $y$ are the horizontal and vertical coordinates of the image, respectively, $\mu(x, y)$ is the gray scale average of $k$ 'th gray normalized exposure image. To avoid getting too small fusion weights from the pixel values of over-bright or over-dark images, we use a weighted method to calculate the expectation of Gauss distribution, which is shown in Eq. (2). $T_{L}$ denotes the exposure threshold value of the pixel weight with Gaussian distribution, usually the range of $T_{L}$ is 0.04 to 0.12 and we set it to 0.1 in this paper. $\sigma$ is standard deviation of the Gaussian distribution. If $0.1<I_{k}^{\text {gray }}(x, y)<0.9, B_{k}(x, y)$ can be calculated by Gauss distribution, whose expectation $\mu(x, y)$ is between 0.35 and 0.65 . To ensure that most of $I_{k}^{\text {gray }}(x, y)$ are in $(\mu-\sigma, \mu+\sigma), \sigma$ should be in $(0.25,0.55)$ and we set it to 0.3 in this paper.

To obtain high-quality 3-D data, the collected images should also contain rich details and edge features. In fact, images contain different details feature richness under different exposure conditions. ${ }^{15}$ Thus image details factor $C_{k}(x, y)$ was selected as another key factor of image fusion. Because the Laplacian has rotation invariance, Laplace filter $L$ is selected to extract the detailed features of $I_{k}^{\text {gray }}(x, y)$, and the detail image $D_{k}(x, y)$ of $I_{k}^{\text {gray }}(x, y)$ are obtained. In order to reduce the image noise, the absolute value of the image after extraction of features was taken and the Gaussian low-pass filter is applied, and then the image detail factor $C_{k}(x, y)$ can be expressed as

$C_{k}(x, y)=H\left(\left|D_{k}(x, y)\right|\right)=e^{\frac{-D_{k}^{2}(x, y)}{2 D_{0}^{2}}}$,

where

$D_{k}(x, y)=L\left[I_{k}^{\text {gray }}(x, y)\right]=\nabla^{2} I_{k}^{\text {gray }}(x, y)$,

with $L$ being the Laplace filter. We have 
$D_{k}(x, y)=\sqrt{\left(x-\frac{P}{2}\right)^{2}+\left(y-\frac{Q}{2}\right)^{2}}$

where $D(x, y)$ denotes the distance between pixel point and frequency center, $D_{0}$ is the Gaussian filter cutoff frequency, to retain as many details as possible in the images, usually the range of $D_{0}$ is 100 to 180 and we set it to 140 in this paper, $P$ is the number of rows of $D_{k}(x, y)$, and $Q$ is the number of columns of $D_{k}(x, y)$.

By considering exposure intensity factor and image detail factor, the fusion weight $\hat{W}_{k}(x, y)$ can be obtained as the product of the two key factors. In order to ensure the sum of the pixel weights is equal to 1, the figure fusion weight $\hat{W}_{k}(x, y)$ is normalized, which is denoted by $\hat{W}_{k}^{\text {norm }}(x, y)$ i.e.,

$\hat{W}_{k}(x, y)=B_{k}(x, y) \times C_{k}(x, y)$,

$\hat{W}_{k}^{\text {norm }}(x, y)=\frac{\hat{W}_{k}(x, y)}{\sum_{m=1}^{N} \hat{W}_{m}(x, y)}$.

The normalized fusion weight $\hat{W}_{k}^{\text {norm }}(x, y)$ often contains noise. Recursive filter is adopted to obtain a refined image $W_{k}^{\text {norm }}(x, y)$ as

$$
\begin{aligned}
W_{k}^{\text {norm }}(x, y) & =\operatorname{RF}\left[\hat{W}_{k}^{\text {norm }}(x, y), \hat{W}_{k-1}^{\text {norm }}(x, y)\right] \\
& =\hat{W}_{k}^{\text {norm }}(x, y)+K\left[\hat{W}_{k-1}^{\text {norm }}(x, y)-\hat{W}_{k}^{\text {norm }}(x, y)\right],
\end{aligned}
$$

where $W_{k-1}^{\text {norm }}(x, y)$ denotes the fusion weight of the previous frame image, $K$ is the filter coefficient, for a better filtering effect, $K$ should be greater than 0.65 , and its value is taken as 0.9 in this paper.

In the end, through the fusion of $N$ images with different exposure times, a high-dynamic range image $E(x, y)$ was obtained as

$E(x, y)=\sum_{k=1}^{N} I_{k}^{\text {gray }}(x, y) \times W_{k}^{\text {norm }}(x, y)$.

\subsection{Adaptive Selection of Image Exposure Time}

A three-wavelength, six-step phase-shift method is used in this work. This method needs to collect 18 images with different wavelengths and phase shifts. If images with different exposure times are collected for fusion, $18 \times$ Nimages are needed in total. Choosing the value of $N$ not only impacts the effectiveness of the fusion, but also affects the real-time performance of 3-D reconstruction process.

In addition, the selection of exposure time directly impacts the dynamic range of fused images and affects the reflection elimination in 3-D reconstruction. To handle the above problems, a method of self-adaptive exposure time selection is proposed.

Adding the images with different exposure times can effectively improve the dynamic range of image fusion, but it also increases the fusion time. Moreover, images with similar exposure time can lead to redundancy in dynamic range. So, choosing appropriate exposure times can improve the real-time performance and the accuracy of the method.

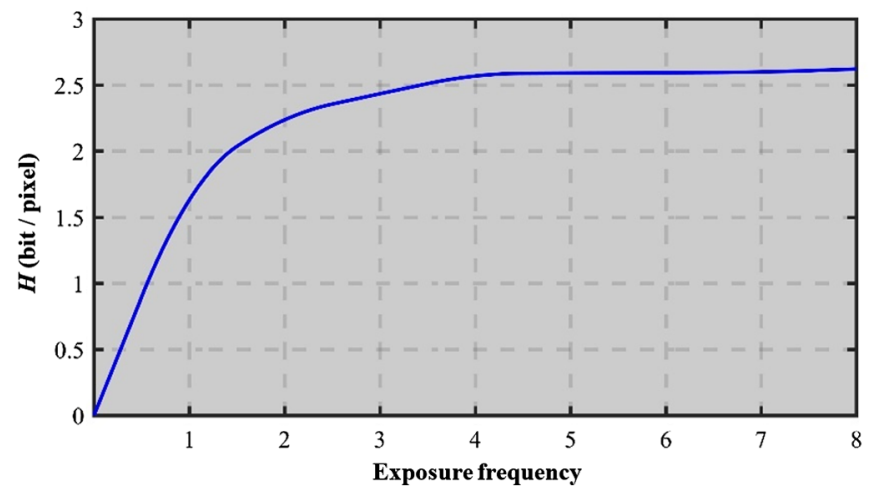

Fig. 2 The relationship between the entropy of image and exposure frequency.

Image information entropy $H$ can effectively measure the amount of information in the image. Therefore, it is often used for measuring image fusion. If $p_{i}$ is the probability of a grayscale, the image information entropy could be represented by

$$
\begin{aligned}
H\left(p_{i}\right) & =\sum_{i=1}^{k} p_{i} * \log \left(\frac{1}{p_{i}}\right) \\
& =p_{1} * \log \left(\frac{1}{p_{1}}\right)+\cdots+p_{k} \log \left(\frac{1}{p_{k}}\right) .
\end{aligned}
$$

Assuming that the exposure time of camera is $t$. When $t<t_{L}$ or $t>t_{H}\left(t_{L}\right.$ and $t_{H}$ are the minimum and maximum effective exposure time), $H$ is $<1$. In order to find the relationship between the information entropy of images and exposures frequency, we fused $N$ images with exposure time $T_{k}=t_{L}+\frac{t_{H}-t_{L}}{N+1} \times k(k=1,2,3, \ldots, N)$ in the same scene, as described in Sec. 2.1, and calculated the information entropy of the fused image. As shown in Fig. 2, when there were more than four exposures, the growth rate of image information entropy slows down. To reduce the operation time of the method under the premise of ensured accuracy, images with four different exposure times are selected for image fusion.

To find the relationship between the information entropy of images and exposure time, we collected four images with exposure time $T_{k}=t_{L}+\frac{t_{H}-t_{L}}{4} \times k(k=1,2,3,4)$ in the same complex scene. For a clearer description, we map $\left[t_{L}, t_{H}\right]$ to $[0,120]$. As shown in Fig. 3, when camera exposure time is in $[0,120]$, the information entropy of the image increases and then decreases with the increase of camera exposure time and a reaches the peak of image information entropy at a specific exposure time. Because four images with different exposure times are needed for image fusion, the exposure time is divided into four intervals, namely, $[0,30],(30,60],(60,90]$, and $(90,120]$. When exposure time is too short, the image information could be missing due to underexposure. However, using dark images can effectively suppress reflection and retain the image details. On the contrary, when the exposure time is too long, overexposure could cause loss of image details, but the image basic profile information could be effectively preserved. So we should select four images in each interval and need to obtain the exposure time of the images with the maximum 


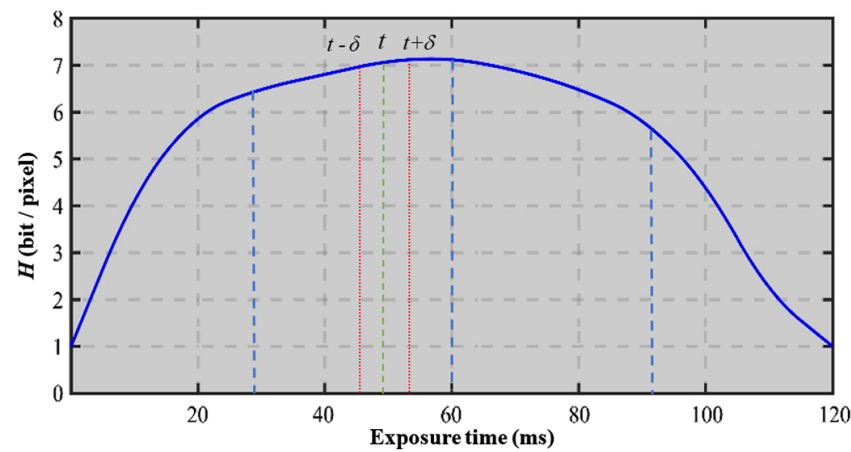

Fig. 3 Adaptive selection of image exposure time, where $\delta \in(5,10)$ and exposure frequency is 4 .

information entropy in each interval. We set the left endpoint as the starting point of the search range, and $\delta$ is the search distance. As shown in Fig. 3, with $(90,120]$ as an example, two kinds of exposure time $(30$ and $30+\delta)$ are adopted, and two exposure images $\left(f_{30}\right.$ and $\left.f_{30+\delta}\right)$ are obtained. If $H f_{30}<H f_{30+\delta}, 30+\delta$ will be selected as the new search endpoint and keep searching the right side until the exposure time corresponded of the interval image information entropy is found, as the best exposure time. Finally, the optimal exposure time $t_{1}, t_{2}, t_{3}$, and $t_{4}$, and the fusion sequences image $I_{k}^{\text {gray }}(x, y)$ of the particular phase under certain frequency are obtained.

\section{High-Dynamic Range 3-D Reconstruction Reflection Suppression Method}

A high-dynamic range image can be obtained through the fusion of images under different exposure times, which solves the problem of missing local image information caused by reflection in the collection image and ensures the integrity of phase information in the reconstruction process. However, the method induces a certain amount of time cost. In order to reduce the processing time in overall reconstruction process and the problem of missing cloud points caused by false matching in the reconstruction process, an improved TWPSP method is adopted in this paper. Assuming the deformation stripes collected by the imaging system is $I_{k}(x, y)$, the six-step phase-shift method adopted in this paper can be described as follows:

$$
\begin{aligned}
& I_{k}(x, y)=A(x, y)+B(x, y) \cos \left[\phi_{i}(x, y)+\frac{\pi \times k}{3}\right], \\
& \quad k=0,1,2,3,4,5
\end{aligned}
$$

where $A(x, y)$ is the background light intensity, $B(x, y)$ is the modulation light intensity, and $\phi_{i}(x, y)$ is wrapped phase, which can be calculated as

$$
\begin{aligned}
& \phi_{i}(x, y)=\arctan \left\{\frac{I_{5}(x, y)-I_{3}(x, y)}{\left[I_{1}(x, y)+I_{4}(x, y)\right]-\left[I_{3}(x, y)+I_{5}(x, y)\right]}\right\}, \\
& i=1,2,3 .
\end{aligned}
$$

The existing TWPSP method ${ }^{16}$ uses $\lambda_{1}=21, \lambda_{2}=18$, and $\lambda_{3}=16$ as the modulation wavelength of projected stripes, which needs to calculate the equivalent wavelength $\lambda_{12}=$ $126, \lambda_{23}=144$, and $\lambda_{123}=1008$ and the corresponding synthetic wrapped phases $\phi_{12}(i, j), \phi_{23}(i, j)$, and $\phi_{123}(i, j)$. Then the corresponding unwrapped phase $\Phi_{3}(i, j)$ corresponding to $\lambda_{3}=16$ can be calculated as

$$
\begin{aligned}
\Phi_{3}(x, y)= & \phi_{3}(x, y)+2 \pi\left\{I N T\left[\frac{\phi_{123}(x, y)}{2 \pi} \times \frac{\lambda_{123}}{\lambda_{23}}\right]\right. \\
& \left.\times \frac{\lambda_{23}}{\lambda_{3}}+\operatorname{INT}\left[\frac{\phi_{23}(x, y)}{2 \pi} \times \frac{\lambda_{23}}{\lambda_{3}}\right]\right\} .
\end{aligned}
$$

The phase unwrapping error caused by the calculation error of $\phi_{12}(i, j), \phi_{23}(i, j)$, and $\phi_{123}(i, j)$ and the multiple jump error of phase unwrapping can result in the missing of 3-D cloud points. In order to avoid the phase error caused by phase synthesis, we select $\lambda_{1}^{\prime}=\lambda_{123}=1008$, $\lambda_{2}^{\prime}=\lambda_{23}=144$, and $\lambda_{3}^{\prime}=\lambda_{3}=16$ as the modulation wavelengths of projected stripes and corresponding wrapped phase $\phi_{i}^{\prime}(x, y)$ can be calculated by Eq. (12), where $\phi_{1}^{\prime}(x, y)=\phi_{123}(x, y), \phi_{2}^{\prime}(x, y)=\phi_{23}(x, y)$, and $\phi_{3}^{\prime}(x, y)=$ $\phi_{3}(x, y)$.

Therefore, the unwrapped phase $\Phi_{3}^{\prime}(x, y)$ corresponding to $\lambda_{3}^{\prime}=16$ can be obtained without calculating the equivalent wavelengths $\lambda_{12}^{\prime}, \lambda_{23}^{\prime}$, and $\lambda_{123}^{\prime}$ of $\lambda_{1}^{\prime}, \lambda_{2}^{\prime}$, and $\lambda_{3}^{\prime}$, and the corresponding synthesized phases $\phi_{12}^{\prime}(x, y), \phi_{23}^{\prime}(i, j)$, and $\phi_{123}^{\prime}(i, j)$. When $\phi_{3}^{\prime}(x, y)=2 \pi$, there is jump in the phase, so the unwrapped phase $\Phi_{3}^{\prime}(x, y)$ is revised to

$$
\Phi_{3}^{\prime}(x, y)=\left\{\begin{array}{l}
\phi_{3}^{\prime}(x, y)+2 \pi\left\{\operatorname{INT}\left[\frac{\phi_{1}^{\prime}(x, y)}{2 \pi} \times \frac{\lambda_{1}^{\prime}}{\lambda_{2}^{\prime}}\right] \times \frac{\lambda_{2}^{\prime}}{\lambda_{3}^{\prime}}+\operatorname{INT}\left[\frac{\phi_{2}^{\prime}(x, y)}{2 \pi} \times \frac{\lambda_{2}^{\prime}}{\lambda_{3}^{\prime}}\right]\right\}, \\
\phi_{1}^{\prime}(x, y) \neq 2 \pi \text { and } \phi_{2}^{\prime}(x, y) \neq 2 \pi \text { and } \phi_{3}^{\prime}(x, y) \neq 2 \pi . \\
\phi_{3}^{\prime}(x, y)+2 \pi\left\{\operatorname{INT}\left[\frac{\phi_{1}^{\prime}(x, y)}{2 \pi} \times \frac{\lambda_{1}^{\prime}}{\lambda_{2}^{\prime}}\right] \times \frac{\lambda_{2}^{\prime}}{\lambda_{3}^{\prime}}+\operatorname{INT}\left[\frac{\phi_{2}^{\prime}(x, y)}{2 \pi} \times \frac{\lambda_{2}^{\prime}}{\lambda_{3}}\right]-1\right\}, \\
\phi_{1}^{\prime}(x, y) \neq 2 \pi \text { and }\left[\phi_{2}^{\prime}(x, y)=2 \pi \text { or } \phi_{3}^{\prime}(x, y)=2 \pi\right] . \\
\phi_{3}^{\prime}(x, y)+2 \pi\left\{\operatorname{INT}\left[\frac{\phi_{1}^{\prime}(x, y)}{2 \pi} \times \frac{\lambda_{1}^{\prime}}{\lambda_{2}^{\prime}}-1\right] \times \frac{\lambda_{2}^{\prime}}{\lambda_{3}^{\prime}}+\operatorname{INT}\left[\frac{\phi_{2}^{\prime}(x, y)}{2 \pi} \times \frac{\lambda_{2}^{\prime}}{\lambda_{3}^{\prime}}\right]\right\}, \\
\phi_{1}^{\prime}(x, y)=2 \pi \text { and } \phi_{2}^{\prime}(x, y) \neq 2 \pi \text { and } \phi_{3}^{\prime}(x, y) \neq 2 \pi .
\end{array}\right.
$$

In this method, $\phi_{1}^{\prime}(x, y)$ and $\phi_{2}^{\prime}(x, y)$ can be directly obtained by Eq. (12), without needing to calculate synthetic phase. Thus it avoids the phase error produced in image fusion, reduces the error in phase unwrapping, and improves the quality of the 3-D point cloud data. The method avoids the phase error caused by the multiple phase synthesis and reduces the time to get the unwrapped phase, thus improving the effectiveness of the proposed method.

The high-dynamic range image obtained in the section is used for the above TWPSP method, which can effectively deal with the reflection problem. An example is shown in Fig. 4. 


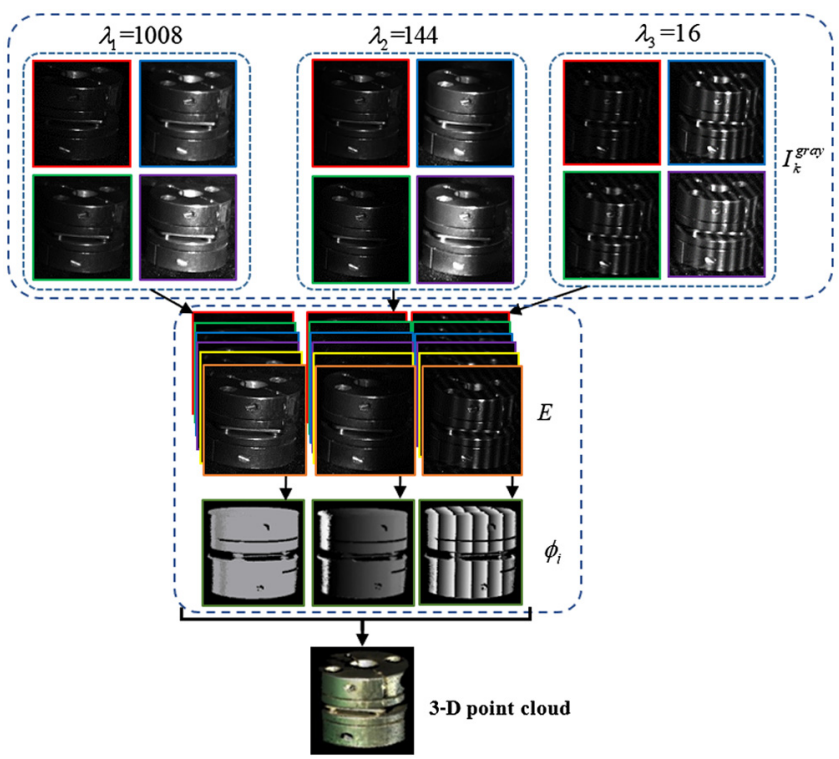

Fig. 4 Reflection suppression method for 3-D measurement.

The use of the MEIF method requires a longer reconstruction time. It is worth mentioning that the improved TWPSP method does not require the calculation of the synthetic phase. For a certain extent, it makes up the time consumed by the whole method, improves the reconstruction accuracy, and accelerates the whole reconstruction process.

\section{Experimental Results and Analysis}

The proposed MEIF was incorporated into our 3-D scanning system (as shown in Fig. 5), consisting of two monochrome (black and white) Gigabit cameras (Microview RS-A1300GM60-M10) with a resolution of $1280 \times 1024$ pixels, one digital projector (LG HW300TC-JE) with a resolution of $1280 \times 800$ pixels, one Gigabit switch (D-Link DGS1005S-CN), and a computer (DELL Optiplex 7010 MT) with a Gigabit Ethernet NIC (Intel 82579LM). Gigabit Ethernet NIC and Gigabit switch are used to control two Gigabit cameras and read the phase-shifting images captured

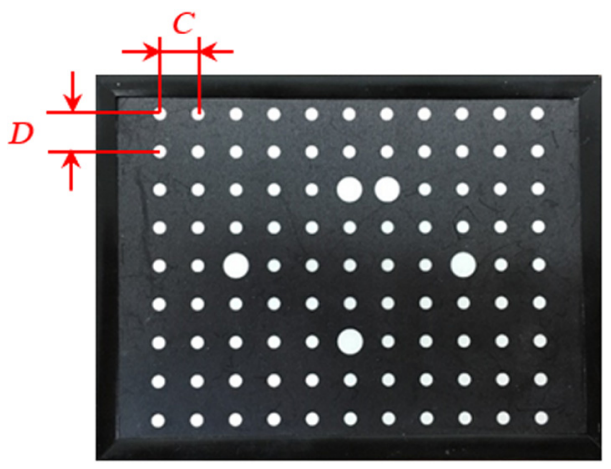

Fig. 6 A plane circular calibration target.

by Gigabit cameras. A workpiece of aluminum alloy with high-reflective surfaces and a workpiece of iron with highreflective surfaces were chosen as the test samples [as shown in Figs. 1(c) and 5]. A plastic shell (as shown in Fig. 8) was measured for analyzing the reconstruction effect of 3-D point cloud using the proposed TWPSP method.

To obtain 3-D reconstruction point data of objects, stereo calibration is carried out on binocular cameras using planar circular calibration target (as shown in Fig. 6). The machining accuracy of the calibration target is $15 \mu \mathrm{m}$, and the true distance between the center of adjacent circles in horizontal direction $C$ is $9 \mathrm{~mm}$, the true distance between the center of adjacent circles in vertical direction $D$ is also $9 \mathrm{~mm}$.

We collected calibration target images from left and right cameras at five different locations, extracted the center of circles in each calibration target image, and used the camera calibration toolbox in OpenCV2.4.11 to get the camera calibration results to calibrate binocular cameras. The principle of this toolbox is based on Zhang's calibration method. ${ }^{17}$ Then the center position of all circles in the calibration target image at each shooting angle [as shown in Figs. 7(a)-7(e)] is obtained by the calibration results.

By measuring the center distance of adjacent circles in horizontal and vertical directions in each calibrated image, we get the error of camera stereo calibration as shown in Table 1 .

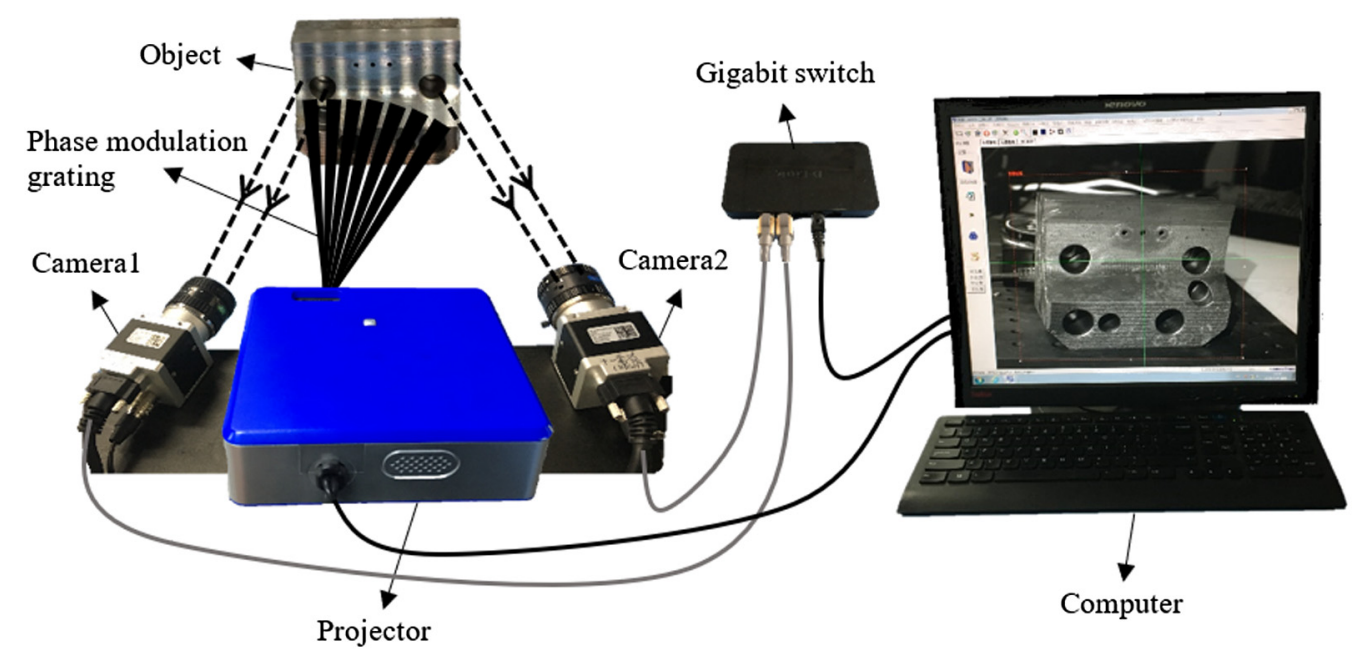

Fig. 5 A 3-D scanning system. 


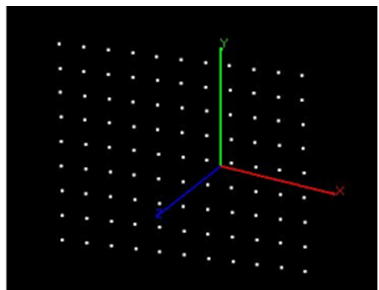

(a)

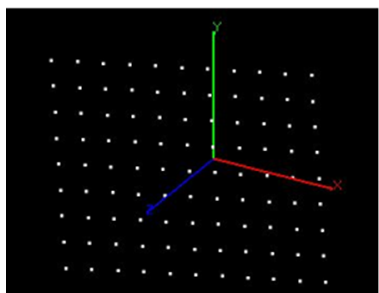

(d)

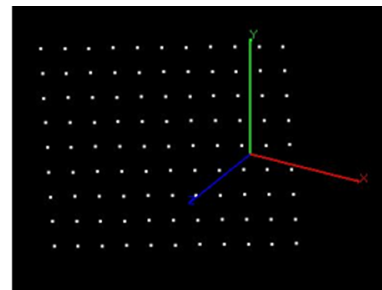

(b)

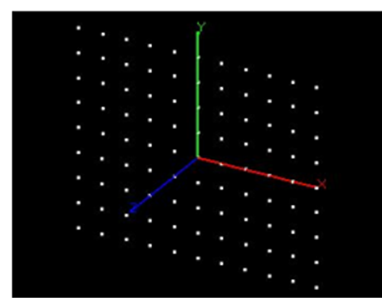

(e)

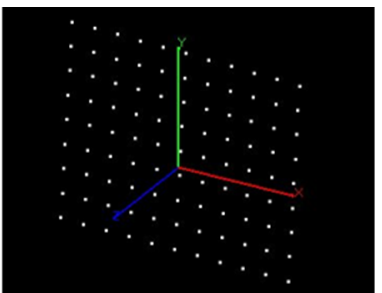

(c)

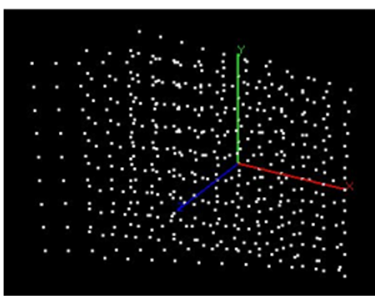

(f)

Fig. 7 The center position of all circles in each calibration target image: (a) first shooting angle, (b) second shooting angle, (c) third shooting angle, (d) fourth shooting angle, (e) fifth shooting angle, and (f) all angles.

Table 1 Root-mean-square error (RMSE) and maximum errors of camera stereo calibration.

\begin{tabular}{lccc}
\hline & $\begin{array}{c}\text { Center distance } \\
\text { between adjacent } \\
\text { circles in horizontal } \\
\text { direction (mm) }\end{array}$ & $\begin{array}{c}\text { Center distance } \\
\text { between adjacent } \\
\text { circles in vertical } \\
\text { direction }(\mathrm{mm})\end{array}$ & $\begin{array}{c}\text { All center } \\
\text { distance } \\
(\mathrm{mm})\end{array}$ \\
\hline RMSE & 0.020 & 0.018 & 0.019 \\
MAX error & 0.047 & 0.059 & 0.059 \\
$\begin{array}{l}\text { (\%) error } \\
\text { (RMSE) }\end{array}$ & 0.222 & 0.200 & 0.211 \\
\hline
\end{tabular}

It can be seen from Table 1 that the RMSE of camera stereo calibration in this paper is $0.019 \mathrm{~mm}$. The largest deviation of the camera stereo calibration is $0.211 \%$, which meets the precise measurement requirements of complex surfaces. Then we use different TWPSP methods to obtain the 3-D data of a plastic shell.

In Fig. 8(a), there are many missing parts of the point cloud data caused by the false matching (the areas selected by red rectangular boxes), but a complete 3-D point cloud data of a plastic packaging shell is shown in Fig. 8(b). Compared to the existing TWPSP method, the improved TWPSP method can effectively reduce the point cloud

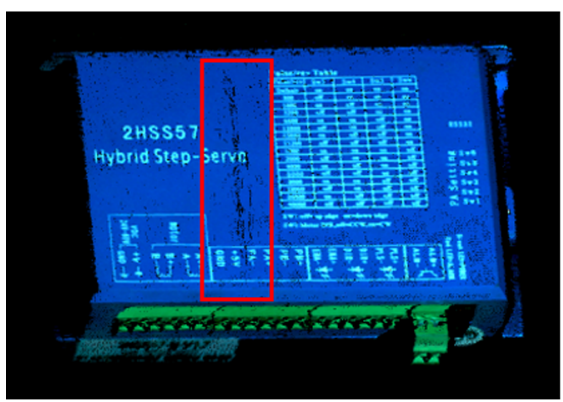

(a)

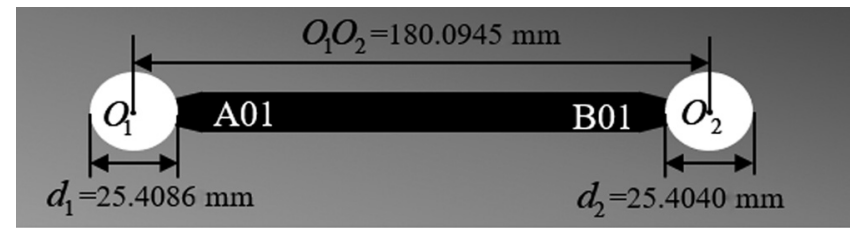

(a)

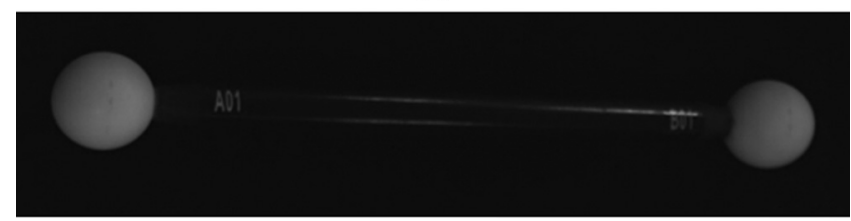

(b)

Fig. 9 Calibration balls used in system measurement accuracy evaluation experiment: (a) dimensions of a calibration ball and (b) a picture of a calibration ball.

data loss caused by the false matching and also achieve more complete point cloud data.

We used the calibration balls shown in Fig. 9, and the measurement scheme is shown in Fig. 10 to obtain the measurement accuracy of 3-D scanning system.

In Fig. 10, the measured scene taken by the camera is divided into five regions ( $\mathrm{I}$ to $\mathrm{V}$ ). The calibration ball is

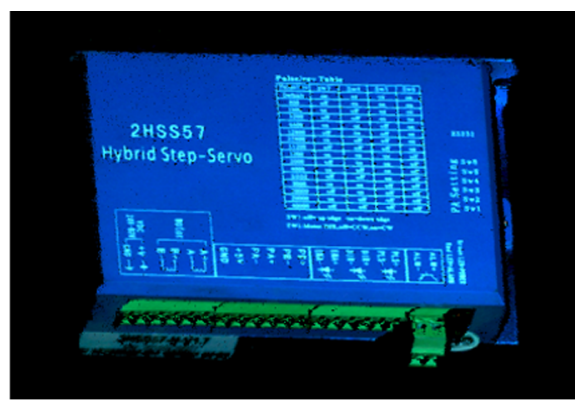

(b)

Fig. 8 3-D reconstruction results of a plastic shell: (a) the 3-D reconstruction result using the existing TWPSP method and (b) the 3-D reconstruction result using the proposed TWPSP method. 


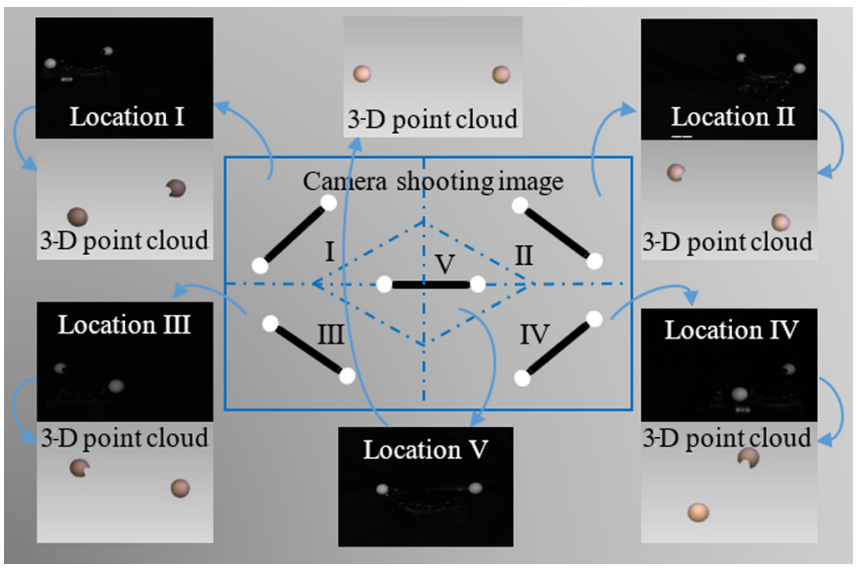

Fig. 10 System measurement accuracy evaluation scheme.

placed in different areas for 3-D scanning to obtain its corresponding 3-D reconstruction data in different areas. Through the measured 3-D data, calibration ball A01 and calibration ball B01 can be fitted, respectively, and obtain the diameters $\hat{d}_{1}$ and $\hat{d}_{2}$ of the two calibration balls, and the sphere centers $\hat{O}_{1}$ and $\hat{O}_{2}$ of the two calibration balls.
Then the distance between the sphere centers of two calibration balls $\hat{O}_{1} \hat{O}_{2}$ can be obtained by the coordinates of $\hat{O}_{1}$ and $\hat{O}_{2}$. We made ten measurements at each location and calculated RMSE of $\hat{d}_{1}, \hat{d}_{2}$, and $\hat{O}_{1} \hat{O}_{2}$ at each location, and the first five measurements and calculations are shown in Table 2.

The average of the RMSE of the $\hat{d}_{1}, \hat{d}_{2}$, and $\hat{O}_{1} \hat{O}_{2}$ measured at different locations is calculated to obtain the measurement accuracy of the system at different locations, and the mean of the measurement accuracy at different locations is taken as the average measurement accuracy of the system. The calculations are shown in Fig. 11.

As shown in Fig. 11, the average measurement accuracy of the system is $0.0598 \mathrm{~mm}$. Compared with other locations, the measurement accuracy of location $\mathrm{V}$ is the highest, which is $0.0278 \mathrm{~mm}$. Therefore, in actual measurement, the object to be measured should be best placed in location $\mathrm{V}$.

In order to verify the effectiveness of the proposed image fusion method, the stripe images were fused under four different exposure times where the projection stripe wavelength $\lambda=144$, and the phase shift angle is $\frac{3}{4} \pi$, which produces the corresponding high-dynamic range images shown in

Table 2 The first five measurements of calibration balls in different positions.

\begin{tabular}{|c|c|c|c|c|c|c|}
\hline Key dimensions & & Location I & Location II & Location III & Location IV & Location V \\
\hline \multirow[t]{6}{*}{$\hat{d}_{1}(\mathrm{~mm})$} & 1 & 25.4692 & 25.3230 & 25.4846 & 25.4574 & 25.4319 \\
\hline & 2 & 25.4562 & 25.4821 & 25.3563 & 25.4699 & 25.4487 \\
\hline & 3 & 25.3469 & 25.4576 & 25.4346 & 25.3516 & 25.4176 \\
\hline & 4 & 25.4740 & 25.4924 & 25.4832 & 25.4718 & 25.4203 \\
\hline & 5 & 25.4928 & 25.4777 & 25.4606 & 25.4658 & 25.4353 \\
\hline & RMSE & 0.0650 & 0.0733 & 0.0591 & 0.0577 & 0.0248 \\
\hline \multirow[t]{6}{*}{$\hat{d}_{2}(\mathrm{~mm})$} & 1 & 25.4734 & 25.3536 & 25.3372 & 25.4692 & 25.4223 \\
\hline & 2 & 25.4890 & 25.4600 & 25.4910 & 25.4514 & 25.4393 \\
\hline & 3 & 25.3536 & 25.4753 & 25.4821 & 25.3219 & 25.4449 \\
\hline & 4 & 25.4938 & 25.4821 & 25.4890 & 25.4706 & 25.4376 \\
\hline & 5 & 25.4692 & 25.4713 & 25.4778 & 25.4530 & 25.4279 \\
\hline & RMSE & 0.0733 & 0.0654 & 0.0785 & 0.0633 & 0.0314 \\
\hline \multirow[t]{6}{*}{$\hat{O}_{1} \hat{O}_{2}(\mathrm{~mm})$} & 1 & 180.0423 & 180.1459 & 180.1803 & 180.1485 & 180.1312 \\
\hline & 2 & 180.1765 & 180.0271 & 180.1669 & 180.1726 & 180.1189 \\
\hline & 3 & 180.0196 & 180.0161 & 180.0116 & 180.1361 & 180.1208 \\
\hline & 4 & 180.1823 & 180.1845 & 180.1465 & 180.1540 & 180.0788 \\
\hline & 5 & 180.1326 & 180.1694 & 180.1715 & 180.1393 & 180.1357 \\
\hline & RMSE & 0.0696 & 0.0735 & 0.0750 & 0.0571 & 0.0303 \\
\hline
\end{tabular}




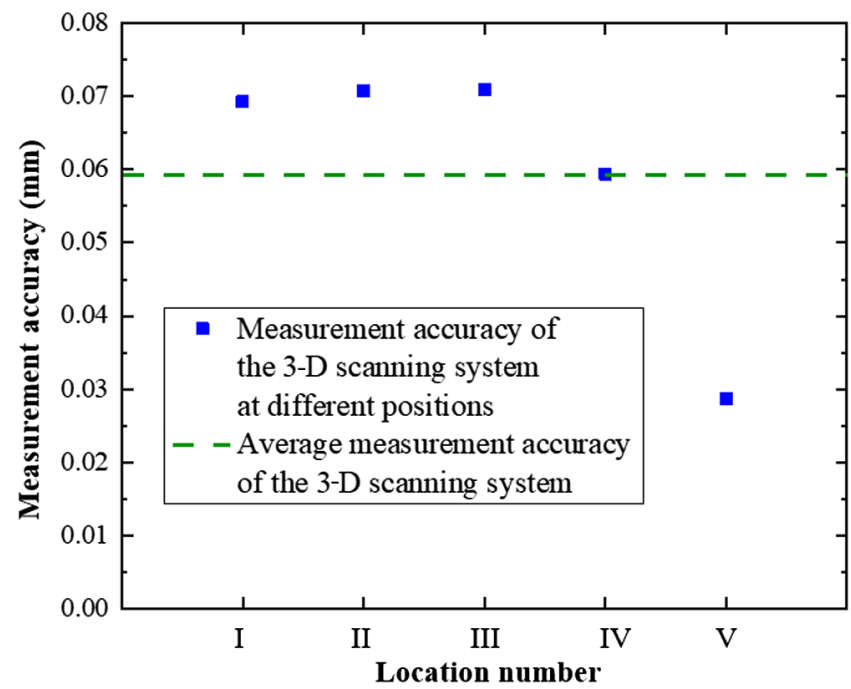

Fig. 11 Measurement accuracy of the system at different positions.

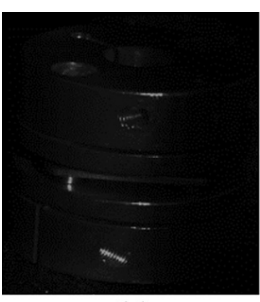

(a)

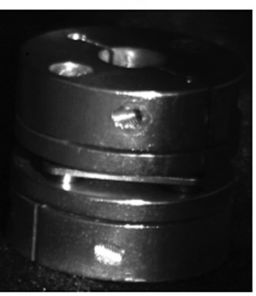

(c)

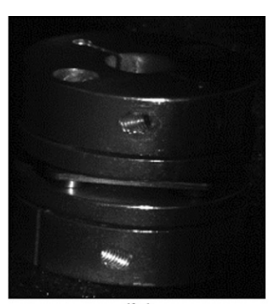

(b)

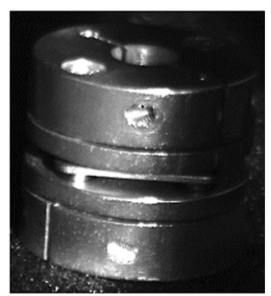

(d)

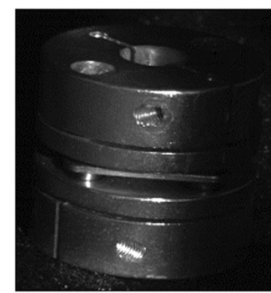

(e)
Fig. 12 Image fusion effect: (a) stripe image with 20-ms exposure time $\left(t_{1}\right)$, (b) stripe image with 52-ms exposure time $\left(t_{2}\right)$, (c) stripe image with 67-ms exposure time $\left(t_{3}\right)$, (d) stripe image with 95-ms exposure time $\left(t_{4}\right)$, and $(\mathrm{e})$ the high-dynamic range image.

Figs. 12(a)-12(d). Figure 12(e) shows the high-dynamic range image obtained through the fusion of the proposed image fusion method. Table 3 compares the image information entropy of corresponding projection stripe images before and after the fusion.

As shown in Fig. 12, the images obtained through the proposed fusion method contains more detailed characteristics, and the fusion image information entropy is improved considerably compared to the images without fusion (as shown in Table 3); and the image information loss problem caused by image overexposure is effectively solved.

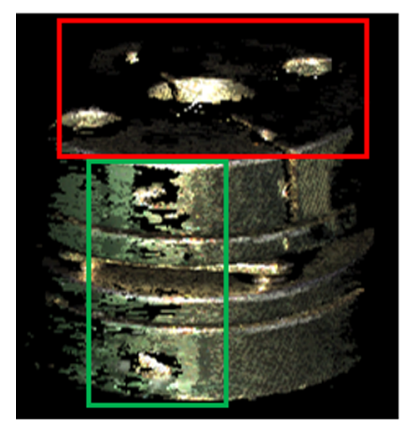

(a)

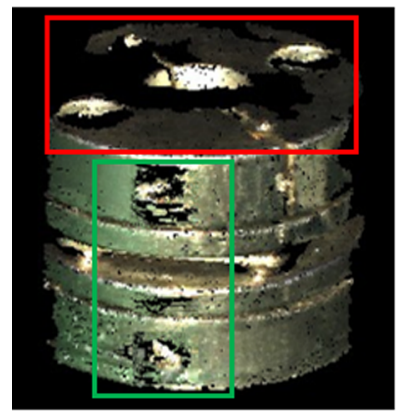

(c)

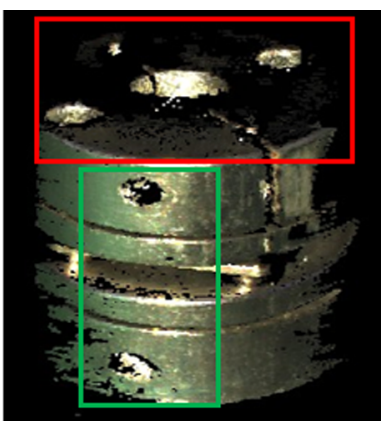

(b)

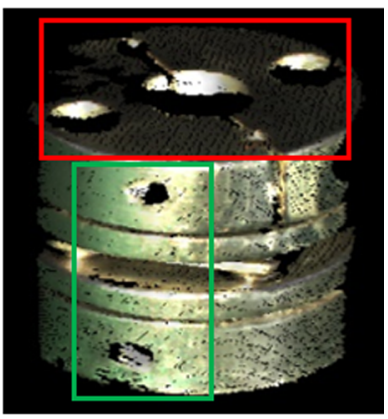

(d)
Fig. 13 Experiment results of an aluminum alloy shaft connector: (a) 3-D reconstruction result using the existing TWPSP method, (b) 3-D reconstruction result from the existing TWPSP methods combined with high-dynamic range images, (c) 3-D reconstruction result using the proposed TWPSP, and (d) 3-D reconstruction result using the reflection suppression method in 3-D measurement of this paper.

To verify the effectiveness of the 3-D measurement reflection suppression method proposed in this paper, the reconstruction performances of the methods using highdynamic range image, the existing TWPSP method, and the improved three-wavelength solution phase method are compared, as shown in Figs. 13 and 14 and Table 4.

In Figs. 13 and 14, the point cloud data selected by the red rectangle boxes are the false matching areas due to the phase unwrapping error, and the point cloud data selected by the green rectangle boxes are the areas where the reflection occurred. The experimental results show that applying highdynamic range image to existing TWPSP method can also achieve more complete point cloud data, and deal with the point cloud loss caused by object reflection. Compared to the traditional three-wavelength solution method, the proposed 3-D measurement reflection suppression method can solve the point cloud loss and point cloud chaos caused by object reflection, also reduce the false matching, get high quality of point cloud data, and thereby achieve effective reflection suppression. However, as seen from Table 4, due to the adopted image fusion method, the whole reconstruction process time is increased. Considering reflection suppression effect and 3-D reconstruction time, the proposed method in

Table 3 Comparisons of image information entropy of stripe images before and after the fusion.

Stripe image with $t_{1} \quad$ Stripe image with $t_{2} \quad$ Stripe image with $t_{3} \quad$ Stripe image with $t_{4} \quad$ The high-dynamic range image

\begin{tabular}{llllll}
\hline$H$ (bit/pixel) & 3.1860 & 3.7157 & 3.9663 & 3.5650 & 4.3749
\end{tabular}




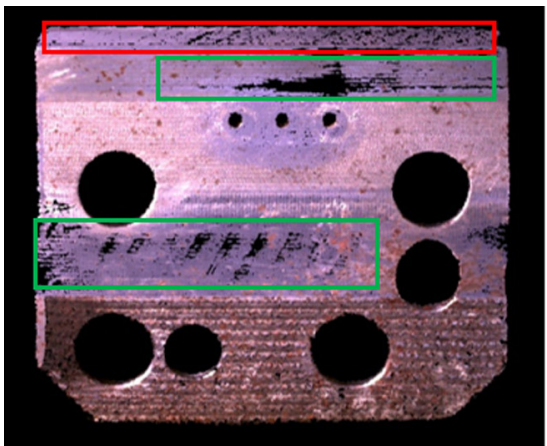

(a)

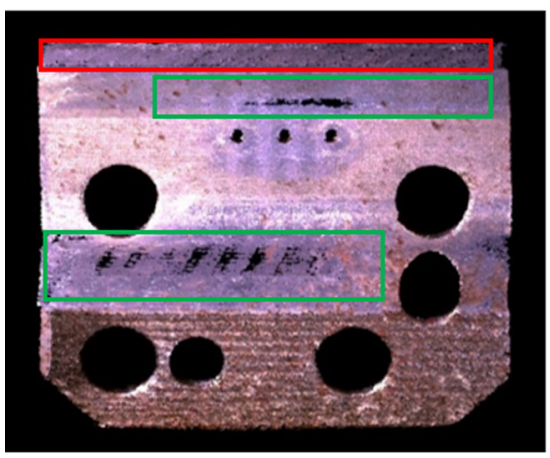

(c)

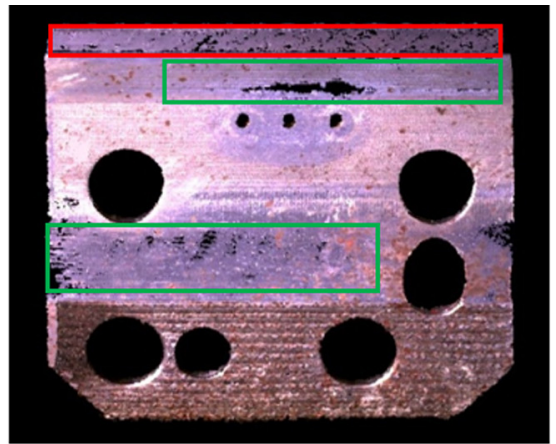

(b)

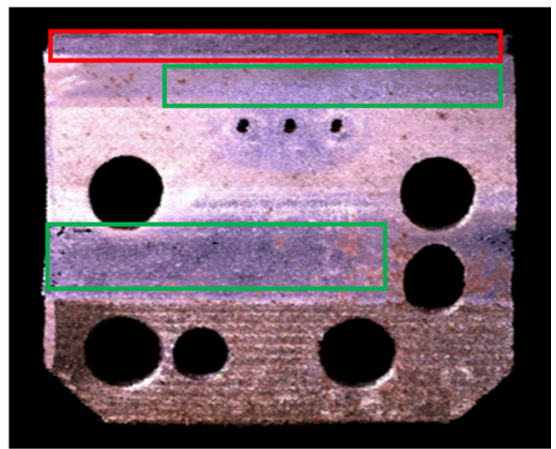

(d)

Fig. 14 Experiment results of an iron mould: (a) 3-D reconstruction result using the existing TWPSP method, (b) 3-D reconstruction result from the existing TWPSP methods combined with high-dynamic range images, (c) 3-D reconstruction result using the proposed TWPSP, and (d) 3-D reconstruction result using the reflection suppression method in 3-D measurement of this paper.

Table 4 Comparison of various methods.

\begin{tabular}{lcccc}
\hline & $\begin{array}{c}\text { The existing } \\
\text { TWPSP method }\end{array}$ & $\begin{array}{c}\text { The existing TWPSP method } \\
\text { combined with MEIF method }\end{array}$ & $\begin{array}{c}\text { The improved } \\
\text { TWPSP method }\end{array}$ & $\begin{array}{c}\text { The proposed reflection } \\
\text { suppression method }\end{array}$ \\
\hline Precision & Low & High & Middle & High \\
Processing speed (s) & 23 & 47 & 12 & 31 \\
Robustness & Low & Middle & Middle & High \\
\hline
\end{tabular}

this paper sacrificed a little time, but it can suppress the reflection and improve the integrity and accuracy of the point clouds.

\section{Conclusion}

In this paper, reflection suppression methods for industrial 3-D measurement have been studied, and a reflection suppression method based on high-dynamic range image obtained by MEIF has been proposed for active 3-D measurement. According to the experimental results, compared to the traditional three-wavelength 3-D measurement method, the proposed reflection suppression method can effectively deal with the point cloud loss and chaos caused by object reflection, and further achieve high accuracy of point cloud data. In addition, compared with the existing reflection suppression methods, the proposed method uses the information entropy of the image to adjust the exposure time adaptively and finally obtains a high-dynamic range phase-shifted image for 3-D reconstruction. However, because the image fusion method in the proposed reflection suppression method needs to traverse all the pixels in images, it may be difficult to achieve real-time 3-D data generation, which is the future improvement of this method.

\section{Acknowledgments}

This research was supported by the National Natural Science Foundation of China (Nos. 61078041 and 51806150) and Natural Science Foundation of Tianjin (Nos. 16JCYBJC15400, 15JCYBJC51700, and 18JCQNJC04400). We thank the National Natural Science Foundation Committee and Tianjin Research Program of Application Foundation and Advanced Technology for the support. This research was also supported by the State Key Laboratory of Precision Measuring Technology and Instruments (Tianjin University) and the Program for Innovative Research Team in University of Tianjin (No. TD13-5036). Moreover, this research was also supported by the Tianjin Enterprise Science and Technology Commissioner Project (No. 18JCTPJC61700). 


\section{References}

1. L. M. Song, Y. Y. Gao, and J. T. Xi, “A 3D measurement method based on multi-view fringe projection by using a turntable," Optoelectron. Lett. 12(05), 389-394 (2016).

2. T. Ebner and O. Schreer, "Fully automated highly accurate 3D reconstruction from multiple views," in IEEE Int. Conf. Image Process., IEEE SigPort (2018).

3. N. M. Garcia, I. de Erausquin, and V. Gruev, "Surface normal reconstruction using circularly polarized light," Opt. Express 23(11), 14391-14406 (2015).

4. Y. Wang et al., "Robust active stereo vision using Kullback-Leibler divergence," IEEE Trans. Pattern Anal. Mach. Intell. 34(3), 548-563 (2012).

5. S. Umeyama and G. Godin, "Separation of diffuses a specular component of surface reflection by use of polarization a statistical analysis of images," IEEE Trans. Pattern Anal. 26(5), 639-647 (2004).

6. C. Waddington and J. Kofman, "Saturation avoidance by adaptive fringe projection in phase-shifting 3D surface-shape measurement," in Int. Symp. Optomechatronic Technol., IEEE, pp. 1-4 (2010).

7. C. Zhang et al.," "A robust surface coding method for optically challenging objects using structured light," IEEE Trans. Autom. Sci. Eng. 11(3), 775-788 (2014)

8. G. Babaie and F. Farahi, "Dynamics range enhancement in digital fringe projection technique," Precis. Eng. 39, 243-251 (2015).

9. G. Babaie, "Dynamic range enhancement imaging in laser interferometry," Meas. Sci. Technol. 26(1), 015202 (2015).

10. S. Zhang and S. T. Yau, "High dynamic range scanning technique," Opt. Eng. 48(3), 033604 (2009).

11. G. H. Liu, X. Y. Liu, and Q. Y. Feng, "3D shape measurement of objects with high dynamic range of surface reflectivity," Appl. Opt. 50(23), 4557-4565 (2011).

12. M. M. Wang, G. L. Du, and Y. J. Li, "Enhanced high dynamic range 3D shape measurement based on generalized phase-shifting method," Opt. Commun. 385, 43-53 (2017).

13. C. Chen, N. Gao, and Z. Zhang, "Adaptive projection intensity adjustment for avoiding saturation in three-dimensional shape measurement," Opt. Commun. 410, 694-702 (2017).

14. J. L. Wu and X. L. Tian, "Image fusion for Mars data using mix of robust PCA," Int. J. Pattern Recognit. 31(1), 1754002 (2017)

15. B. Yang and G. Huang, "Efficient image fusion with approximate sparse representation," Int. J. Wavelets Multi. 14(4), 181-189 (2016).

16. L. M. Song et al., "A new phase unwrapping algorithm based on three wavelength phase shift profilometry method," Opt. Laser Technol. 45 (1), 319-329 (2013).

17. Z. Y. Zhang, "A flexible new technique for camera calibration," IEEE Trans. Pattern Anal. 22(11), 1330-1334 (2000).

Limei Song received her $\mathrm{BE}, \mathrm{ME}$, and $\mathrm{PhD}$ degrees from Tianjin University, Tianjin, China, in 1999, 2001, and 2004, respectively, all in measurement technology and instrumentation. She is a professor at Tianjin Polytechnic University. She is the author of more than 80 journal papers and has written 3 book chapters. Her research interests include machine vision, 3-D optical measurement, reverse engineering, and pattern recognition. She is a member of SPIE.

Yuan Ru received his BE degree from Tianjin Polytechnic University, Tianjin, China, in 2012, in automation. He is a student who is studying for his ME degree at Tianjin Polytechnic University, Tianjin, China. His research interests include machine vision, 3-D optical measurement, pattern recognition, and artificial intelligence.

Yangang Yang received his $\mathrm{BE}$ and $\mathrm{ME}$ degrees from Tianjin University, Tianjin, China, in 1999, 2004, all in measurement technology and instrumentation. He was CTO of Tianjin Tong Yang Company and the leader of the National Special Project-Online Automatic Monitoring of Odor. He is a teacher at Tianjin University of Technology and Education. His research interests include machine vision, optical spectrum analysis, and reverse engineering.

Qinghua Guo received his BE degree in electronic engineering and his ME degree in signal and information processing from Xidian University, Xi'an, China, in 2001 and 2004, respectively, and his $\mathrm{PhD}$ in electronic engineering from the City University of Hong Kong, Kowloon, Hong Kong, in 2008. He is currently in the School of Electrical, Computer, and Telecommunications Engineering at the University of Wollongong and also in the School of Electrical Engineering and Automation at Tianjin Polytechnic University. His research interests include signal processing, 3-D reconstruction, and telecommunications.

Xinjun Zhu received his BE degree from Linyi University in 2008, his ME degree from Shandong University of Technology in 2011, and his PhD from Tianjin University in 2015. He joined Tianjin Polytechnic University in 2015. His research interests include fringe analysis, phase retrieval for optical 3-D measurement, and light scattering techniques for particles.

Jiangtao $\mathbf{X i}$ received his $\mathrm{BE}$ degree from Beijing Institute of Technology, Beijing, China, in 1982, his ME degree from Tsinghua University, Beijing, China, in 1985, and his PhD from the University of Wollongong, Wollongong, Australia, in 1996, all in electrical engineering. $\mathrm{He}$ is a professor and the head of School of Electrical, Computer, and Telecommunications Engineering. His research interests include signal processing and its applications in various areas, such as optoelectronics, instrumentation and measurement, and telecommunications. 